\title{
Pulsatile Vs Non-Pulsatile Intracranial Blood Flow: Animal Model of Blood Flow Restoration in Brain Tamponade
}

\author{
Federico Bianchi ${ }^{1 *}$, Carmelo Anile ${ }^{2,3}$, Gianpaolo Petrella ${ }^{4}$, Antonio Ficola ${ }^{5}$ and Michelangelo \\ Iannone $^{6}$ \\ ${ }^{1}$ Pediatric Neurosurgery, Fondazione Policlinico Gemelli IRCCS, Italy \\ ${ }^{2}$ Neurosurgery Fondazione Policlinico Gemelli IRCCS, Italy \\ ${ }^{3}$ Catholic University of the Sacred Heart, Italy \\ ${ }^{4}$ Ospedale Santa Maria Goretti, Italy \\ ${ }^{5}$ Department of engineering, University of Perugia, Italy \\ ${ }^{6}$ CNR staff chief, secondary office in Roccelletta di Borgia (CZ), Italy
}

*Corresponding author: Bianchi F, Fondazione Policlinico Gemelli IRCCS, Largo Agostino Gemelli 8, 00168 Rome (RM), Italy

\section{ARTICLE INFO}

Received: 幽 September 20, 2021

Published: 彗 September 27, 2021

Citation: Federico Bianchi, Carmelo Anile, Gianpaolo Petrella, Antonio Ficola, Michelangelo Iannone. Pulsatile Vs Non-Pulsatile Intracranial Blood Flow: Animal Model of Blood Flow Restoration in Brain Tamponade. Biomed J Sci \& Tech Res 39(1)-2021. BJSTR. MS.ID.006240.

Keyword: Experiment; Pressure; Tamponade; Pulsatile

Abbreviations: ICP: Intracranial Pressure; SAP: Systemic Arterial Blood Pressure; CAP: Carotid Arterial Blood Pressure; CPP: Cerebral Perfusion Pressure; CBF: Cerebral Blood Flow
ABSTRACT

Brain tamponade represents the end-line for neurosurgical treatment. In order to overcome such an obstacle, the authors present an animal model in which changing the modalities of brain blood supply a certain degree of maintained cerebral perfusion is possible even in conditions of highly elevated intracranial pressure. Five sheep were surgically prepared to evaluate tamponade brain in:

1) Normal condition

2) Continuous laminar flow created using extracorporeal circulation

3) Combined model. In this model the brain is submitted to pulsatile circulation created with the aforementioned pulsatile machine in EC switching in a second moment to continuous flow in order to evaluate differential response to flow modifications.

Normally, whenever there is an increase in intracranial pressure, the difference in transient propagation speed lead to an early closure of the veins and of the Starling resistor before intravasal pressure could match outer one maintaining positive flow. On the other hand, if the circulation were non-pulsatile a net flow would be always present thanks to the persistence of pressure gradient. Our data might represent the first step in that direction showing how changing cerebral flow even tamponade can temporarily overcome. Even though this is only an animal experiment it might open the way to further animal experiment and thus to human ones.

\section{Introduction}

In neurosurgical practice brain tamponade represents the ultimate limit for treatment. It is defined as a progressive intracranial pressure (ICP) increase up to values close to arterial blood pressure producing a reverberating flow pattern in the cerebral arteries with no net flow [1-3]. Nowadays, patients reaching such a condition are labeled as untreatable due to the lack of effective treatment. Decompressive craniectomy might in peculiar conditions, such as in very little children, overcome the aforementioned limit thanks 
to the incredible capability of a still growing brain to recover from extensive injuries but that is not the case in adult or elder patient. Throughout the literature there are several papers addressing the matter but still no clear advance was proposed. In fact, many of the papers are still at the animal levels also due to the actual difficulties in creating an ethically approvable human model. This aspect is linked to the fact the patients near brain tamponade conditions have to be rapidly treated whenever possible being hard to create a double group-controlled study. Furthermore, it is not so easy to define the actual limit in which brain tamponade become irreversible. The authors themselves, in previous papers, highlighted how even in prolonged brain tamponade conditions, metabolism inside the neuronal cells still continue even after prolonged ischemia time $[4,5]$.

The idea of overcoming the blockage in cerebral blood flow modifying its modality derived from a previous report of residual arterial and venous pulsation even in tamponade brains [1]. In order to do so, we hereby present an animal model in which changing the modalities of brain blood supply from pulsatile to continuous it might be possible to maintain cerebral perfusion even in conditions of highly elevated intracranial pressure.

\section{Material and Methods}

Five male sheeps (30-35 Kg) were sedated using intramuscular Atropine (0,5-1 mg) and Ketamine (10 mg/Kg). Each animal was placed supine on the operating table, intubated and anesthetized with Halothane (0,8-1\%) and Pancuronium Bromide $(0,5 \mathrm{mg} / \mathrm{h}$ intravenously administrated). These sheeps were evaluated for the whole duration of the procedure using:

a) Electrocardiogram

b) Systemic arterial bold pressure (measured through a line in the obturator artery) (SAP)

c) Carotid arterial blood pressure (CAP)

d) Middle cerebral artery blood flow measured using doppler ultrasound (placed to an ad-hoc craniotomic window)

e) ICP measured using an intra-parenchymal sensor (ICP Express Codman) placed using a parietal burr hole.

Once sedated each animal was prepared in the following way. Two inguinal incisions were made to isolate the femoral arteries that were exposed trough blunt dissection and cannulated. Similarly, through a neck midline incision the carotid arteries were found and prepared. In the meanwhile, a hydraulic circuit was created to ensure extracorporeal circulation. Such a circuit was composed by sylastic tubes, a peristaltic pump, a three-liter reservoir placed at 3-meter height from the ground and lastly from a mechanism granting pulsatility in order to mimic cardiac output. This mechanism is composed by an electric engine connected to a piston compressing the elastic portion of the tube exiting the reservoir. By doing so modifying the compression speed and the distance of the piston from the tube is possible to modify pulsation frequency and amplitude. The described circuit has three terminals, one for each femoral artery and the remaining one for the left carotid artery (the terminal ends with a Y connector). The whole circuit is replenished before starting with saline solution added with 25000 unit of heparin in order to avoid clotting inside it. To avoid animal hypovolemic state, the reservoir is filled with a liter of saline solution before starting.

To create a condition of intracranial hypertension saline solution will be sent into the subdural space using a 20 Gauge needle inserted through a small, angulated burr hole which is also sealed with acrylic resin in order not to let the fluid escape around the needle. Infusion flow speed was regulated according to the parameter measure by the intra-parenchymal sensor. After clamping of the brachiocephalic trunk, the circuit can be activated. Whenever doing so, the blood taken from the femoral arteries is aspirated and carried into the reservoir from where, thanks to gravity, it flows into the left carotid artery. Thanks to the Y connector the blood in the left carotid artery can flow both toward the brain and towards the base of the brachiocephalic trunk granting blood supply to the whole brachiocephalic territory. The pulsation machine intervenes in this setting in order to transform a pulsatile flow into a continuous one without creating relevant changes in medium arterial pressure. Once completed animal preparation, three different experimental conditions were evaluated in order to measure the cerebral perfusion pressure ( $\mathrm{CPP}=\mathrm{CAP}-\mathrm{ICP})$ value at which cerebral blood flow (CBF) blockage appear in each of them. The aforementioned conditions are:

a) Normal condition

b) Continuous laminar flow created using EC

c) Combined model. In this model the brain is submitted to pulsatile circulation created with the aforementioned pulsatile machine in EC switching in a second moment to continuous flow in order to evaluate differential response to flow modifications.

At the end of the experiment the animals were sacrificed being still under general anesthesia using an intravenous administration of $10 \mathrm{mEq}$ potassium chloride. The whole experiment was carried on in accordance with the EU Directive 2010/63/EU for animal experiments.

\section{Results}

A. Model 1: mean CAP value is $110 \mathrm{mmHg}$ (ranging from 100 to $130 \mathrm{mmHg}$ ) while mean ICP value is $15 \mathrm{mmHg}$ (ranging from 12 to $18 \mathrm{mmHg}$ ) and mean MCA speed is about $10 \mathrm{~cm} / \mathrm{sec}$. Starting saline subdural infusion, ICP value start increasing while CBF progressively decrease. This process continues until ICP 
reaches $70 \mathrm{mmHg}$ with consequential $\mathrm{CBF}$ blockage. Even though no blood flow can be measured at this moment, CPP is still present and greater than $40 \mathrm{mmHg}$. At the same moment, a different behavior of CBF velocity can be observed. In fact, even if CPP is still present flow velocity reaches zero concomitantly wit tamponade. Both observations tend to recover baseline condition once stopped infusion.

B. Model 2: the initial increase in ICP and decrease in CBF speed is the same of model 1 but, unlike with pulsatile flow, CBF arrest is reached with higher ICP value. In fact, ICP values similar to CAP are needed in this case with a residual CPP of 15-16 $\mathrm{mmHg}$ to observe cerebral tamponade. The observation concerning CBF speed overlaps what seen in model 1 . As in model 1 this condition is reversible after infusion arrest.

C. Model 3: the combined model shows firstly how normal cardiac circulation can be achieved using pulsatile EC with similar results on CPP and CBF speed. On the other hand, it shows how, switching from pulsatile to continuous flow in absence of relevant changes in CPP, a gradual and stable intracranial circulation can be obtained as documented by doppler ultrasound. The aforementioned results are summarized in Figure 1.

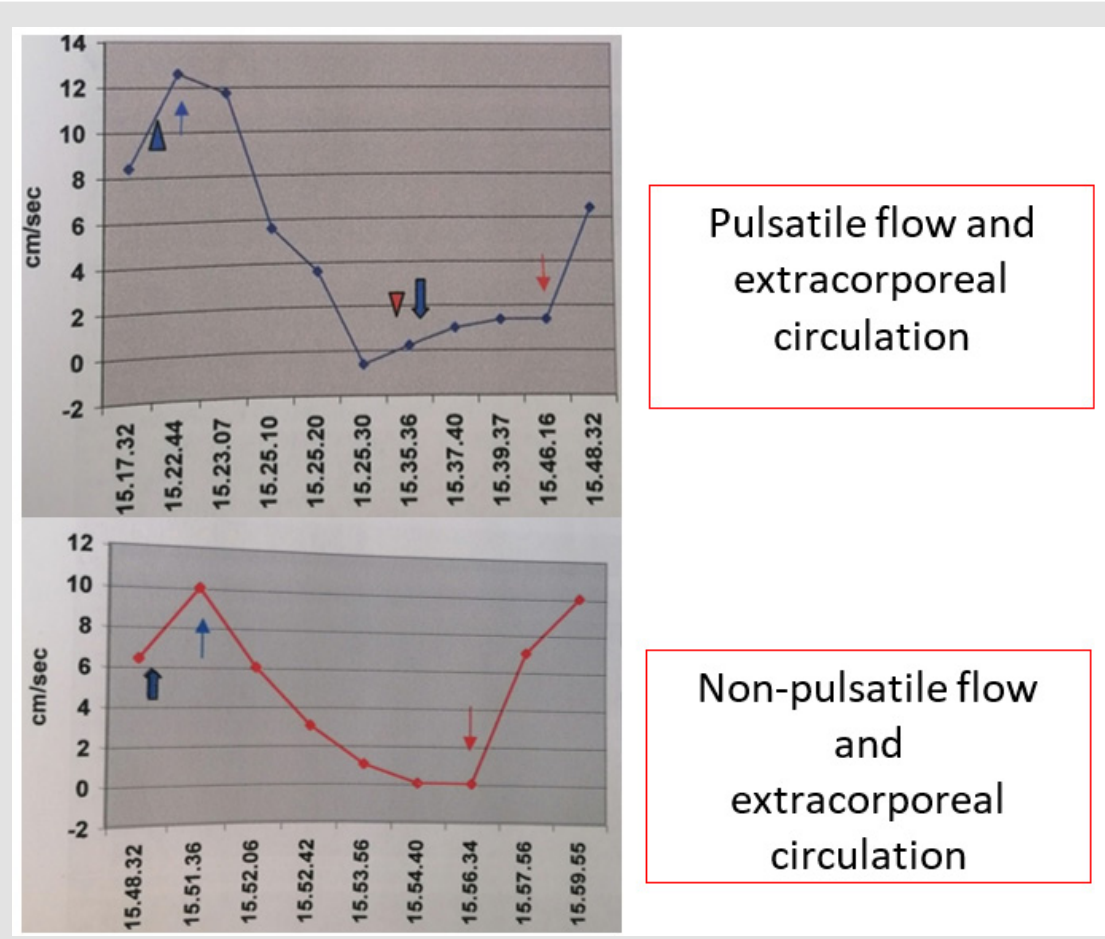

Figure 1: Blood flow velocity in different circulations.

\section{Discussion}

Throughout the literature, there are very few reports regarding flow typology in intracranial circulation. Such papers are mostly related to intracranial changes after ischemic heart failure. Reviewing the literature trying to select the most fitting papers, only two authors slightly address the problem. the first one only mentions non pulsatile blood flow as something unclear as well as a potential sign for proximal arterial occlusion [6], while the other one, suggests the importance of pulsatile flow during reperfusion without addressing at all flow modifications during tamponade [7]. To overcome such a lack of evidence on the matter, the authors devised the presented experiment. The aim was to analyze whether changing cerebral blood flow from pulsatile to non-pulsatile was possible to overcome brain tamponade. Such an experiment was founded on the idea that the very "normal" blood pulsation coupled with Starling resistor functioning is at the base of cerebral tamponade. Physical laws states that flow is driven by the presence of a pressure gradient between two compartments connected by a channel. Thus, as long there is a gradient there will be flow, no matter how small the caliber of the channel will become. Flow stops then after the closure of the channel or after disappearance of the gradient. The application of such physical law to the intracranial system were evaluated for the first time by Chopp et al. who created a model simulating the intracranial space and its modifications during infusion tests [8].

In order to describe what happen in normal conditions, it is important to remember that intracranial circulation is pulsatile and that pressure wave propagation speed inside the vascular system is slower than the liquoral one due to the resistance in capillaries and veins. Thus, whenever there is an increase in 
intracranial pressure, the aforementioned difference in transient propagation speed lead to an early closure of the veins and of the Starling resistor before intravasal pressure could match outer one maintaining positive flow. When the vein walls contact each other the possibility to re-open is lost leading to tamponade. On the other hand, if the circulation were non-pulsatile a net flow would be always present thanks to the persistence of pressure gradient. Such persistence is granted by the absence of a pulsation wave preventing the previously described vein closure mechanism. The channels will become smaller in an asymptotic way never actually closing and preventing the reach of zero net flow. Obviously, this situation is theoretical and in reality, the channels will eventually close, but a greater intracranial pressure would be needed. In order to demonstrate such an assumption, we have created a model of selective extracorporeal brachiocephalic circulation in order to send laminar flow to the brain without affecting body circulation.

The selection of the sheep as animal model was made in order to simplify the experiment having this animal a peculiar anatomy of the brachiocephalic trunk. In fact, in this setting all of the vessels, emerging in the human from the aortic arch, start from this trunk. From left to right it emerges first the left subclavian artery the two carotid arteries and last the right subclavian artery. Such conformation simplifies the experiment granting the selectivity control of the cerebral blood flow through the manipulation of a single vessel. Nonetheless, it is important to remember that collateral circulation might be present in selected cases reducing the power of the experimental model. In the sheep model though, such collaterals disperse most of their contribution to the spinal roots and to the neck muscle making the amount of cerebral distribution negligible. Dividing the experiment into three moments granted us the possibility not to miss biases in the model. In model 1 the authors confirmed a similar trend between sheeps and humans regarding brain tamponade. Blood flow ceases concomitantly with an increase of ICP over CPP reaching brain tamponade even in condition of persistent low CPP. Model 2 differs from model 1 in the need for a higher ICP value to reach tamponade and flow absence. Such a finding suggests a higher threshold to be reached in order to cause it. Finally, model 3 unites the previous ones and improves them showing how a change in flow type might overcome a preexisting tamponade situation offering a possible novel treatment strategy. The most striking data reside in the reappearance of blood flow during tamponade after the change from pulsatile flow to continuous one.

\section{Conclusion}

Brain tamponade in neurosurgery represents nowadays the terminal line for treatment. Every effort has to be made in order to find a way to overcome such a limit. Our data might represent the first step in that direction showing how changing cerebral flow even tamponade can temporarily overcome. Even though this is only an animal experiment it might open the way to further animal experiment and thus to human ones.

\section{Funding}

This research did not receive any specific grant from funding agencies in the public, commercial, or not-for-profit sectors.

\section{Declaration of Interest}

None.

\section{Acknowledgments}

None.

\section{References}

1. Carmelo A, Ficola A, Fravolini ML, La Cava M, Maira G, et al. (2002) ICP and CBF regulation: a new hypothesis to explain the "windkessel" phenomenon. Acta Neurochir 81: 112-116.

2. Lindegaard KF, Grip A, Nornes H (1980) Precerebral haemodynamics in brain tamponade. Part 1: Clinical studies on blood flow velocity. Neurochirurgia (Stuttg) 23(4): 133-142.

3. Lindegaard KF, Grip A, Nornes H (1980) Precerebral haemodynamics in brain tamponade. Part 2: Experimental studies. Neurochirurgia (Stuttg) 23(5): 187-196.

4. Bianchi F, Di Muro Licia, Petrella Gianpaolo, Rigante Luigi, Anile Carmelo, et al. (2020) Metabolic Changes During and After Global Prolonged Reversible Cerebral Ischemia in Pigs. Is it Possible to Change Brain Metabolism to Maintain the Brain Alive? Biomed J Sci Tech Res 25: 18939-18946.

5. Vrselja Z, Daniele SG, Silbereis J, Talpo F, Morozov YM, et al. (2019) Restoration of brain circulation and cellular functions hours postmortem. Nature 568: 336-343.

6. Kim YS, Chernyshev OY, Alexandrov AV (2006) Nonpulsatile cerebral perfusion in patient with acute neurological deficits. Stroke 37(6): 15621564

7. Allen BS, Ko Y, Buckberg GD, Tan Z (2012) Studies of isolated global brain ischaemia: III. Influence of pulsatile flow during cerebral perfusion and its link to consistent full neurological recovery with controlled reperfusion following $30 \mathrm{~min}$ of global brain ischaemia. Eur J CardioThorac Surg Off J Eur Assoc Cardio-Thorac Surg 41(5): 1155-1163.

8. Chopp M, Portnoy HD, Branch C (1983) Hydraulic model of the cerebrovascular bed: an aid to understanding the volume-pressure test. Neurosurgery 13(1): 5-11. 
ISSN: 2574-1241

DOI: $10.26717 /$ BJSTR.2021.39.006240

Federico Bianchi. Biomed J Sci \& Tech Res

(c) (P) This work is licensed under Creative Commons Attribution 4.0 Licens

Submission Link: https://biomedres.us/submit-manuscript.php

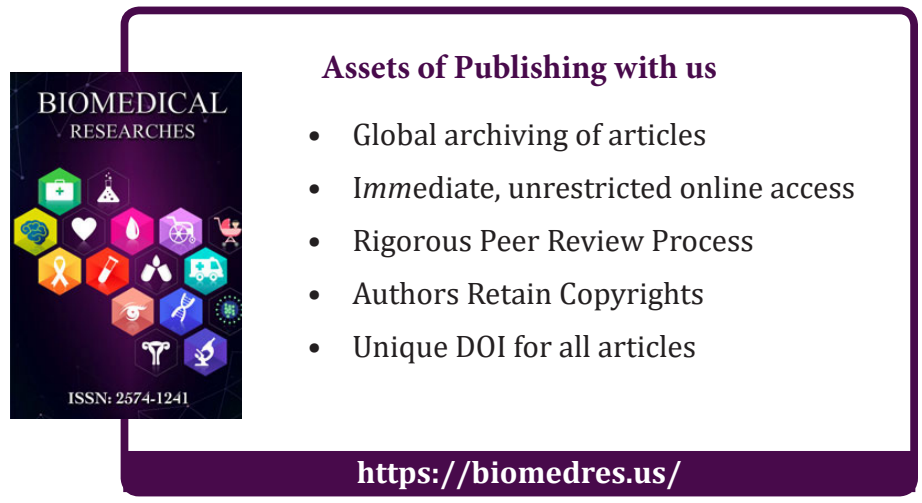

